\section{On Sparse Representations in Arbitrary Redundant Bases}

Jean-Jacques Fuchs, Member, IEEE

\begin{abstract}
The purpose of this contribution is to generalize some recent results on sparse representations of signals in redundant bases. The question that is considered is the following: given a matrix $A$ of dimension $(n, m)$ with $m>n$ and a vector $b=A x$, find a sufficient condition for $b$ to have a unique sparsest representation $x$ as a linear combination of columns of $A$.

Answers to this question are known when $A$ is the concatenation of two unitary matrices and either an extensive combinatorial search is performed or a linear program is solved. We consider arbitrary $\boldsymbol{A}$ matrices and give a sufficient condition for the unique sparsest solution to be the unique solution to both a linear program or a parametrized quadratic program. The proof is elementary and the possibility of using a quadratic program opens perspectives to the case where $b=A x+e$ with $e$ a vector of noise or modeling errors.
\end{abstract}

Index Terms-Basis pursuit, global matched filter, linear program, quadratic program, redundant dictionaries, sparse representations.

\section{INTRODUCTION}

Let us consider a set of $m n$-dimensional vectors $a_{j}$ with $m>n$ and let us denote $A$ the $(n, m)$ matrix having these vectors as columns. Any linear combination $b$ of these $m$ vectors can then be written as $b=A x$ with $x$ an $m$-dimensional vector of weights.

If $x$ has just a few nonzero components it may well be the unique and sparsest representation and to recover it from the knowledge of $b$ one could then seek the sparsest among all the solutions to $A x=b$. The aim of this contribution is to give conditions under which this is feasible in reasonable time.

Finding the solution having the smallest possible number of nonzero components, i.e., solving the following optimization problem:

$$
\min _{x}\|x\|_{0} \text { subject to } A x=b
$$

where $\|x\|_{0}$ denotes the number of nonzero components in $x$, is a difficult problem that can only be solved using a combinatorial approach, i.e., testing systematically all the potential combinations of columns. This approach is thus unfeasible and it is usual to consider instead the following much simpler optimization problem:

$$
\min _{x}\|x\|_{1} \text { subject to } A x=b
$$

where $\|x\|_{1}=\sum\left|x_{i}\right|$ denotes the $\ell_{1}$ norm of $x$. The problem (LP) is easily transformed into a linear program whose solution is straightforward to obtain.

The case where $A$ is the concatenation of two square orthogonal matrices $U_{1}$ and $U_{2}: A=\left[\begin{array}{ll}U_{1} & U_{2}\end{array}\right]$, was investigated by Donoho and Huo [1] and Elad and Bruckstein [2]. It was shown that if a solution $x$ satisfies

$$
\|x\|_{0}<\frac{1}{M}, \quad \text { with } M=\sup _{1 \leq i \neq j \leq m}\left|a_{i}^{T} a_{j}\right|
$$

Manuscript received December 5, 2002; revised February 13, 2004. The material in this correspondence was presented in part at the 13th IFAC Symposium on Identification and System Parameter Estimation (SYSID), Rotterdam, The Netherlands, August 2003.

The author is with the IRISA/Université de Rennes I, Campus de Beaulieu, F-35042 Rennes Cedex, France (e-mail: fuchs@irisa.fr).

Communicated by G. Battail, Associate Editor At Large.

Digital Object Identifier 10.1109/TIT.2004.828141 then it is the unique sparsest solution and that under the stronger condition

$$
\|x\|_{0}<\frac{1}{2}\left(1+\frac{1}{M}\right), \quad \text { with } M \text { as above }
$$

the unique sparsest solution is also the unique minimum point of (LP). Stronger results are established in [2] for this kind of $A$ matrices.

In the following, we prove that if condition (2) is satisfied by a solution $x_{o}$ of $A x=b$, with $A$ a matrix built upon an arbitrary number of vectors with unit Euclidean norm, then it is the unique minimum point of both a parametrized quadratic program (QP) in a sense to be defined later (Theorems 2 and 3), and the linear program (LP) (Theorems 3 and 4). But this implies that $x_{o}$ is also the the unique sparsest solution of $A x=b$ since if a sparser representation existed, it would satisfy (2), the same reasoning would hold and one would arrive at a contradiction since both (QP) and (LP) are convex programs that have a unique optimum attained at a single point under (2). A similar result has independently been obtained in [3] for (LP) using a completely different approach.

To establish this result, we merely apply more general but nonexplicit results presented in [4], [5] to this very specific problem where the emphasis is on sparsity. The proof we present goes through if the vectors $a_{j}$ are not normalized but its last part (see Section V) would be more intricate.

\section{THE CRITERION}

Instead of (LP), let us consider the following optimization problem:

$$
\min _{x} \frac{1}{2}\|A x-b\|_{2}^{2}+h\|x\|_{1}, \quad h>0
$$

where $\|x\|_{2}^{2}=\left(\sum x_{i}^{2}\right)$ denotes the $\ell_{2}$ norm of $x$. This is a convex unconstrained nonsmooth optimization problem that can be converted into a (QP) [6]. Introducing the new variables $x_{i}^{+}=\max \left(x_{i}, 0\right), x_{i}^{-}=$ $\max \left(-x_{i}, 0\right)$ and constraining them to be greater than or equal to zero, one can then replace $x_{i}$ by $x_{i}^{+}-x_{i}^{-}$and $\left|x_{i}\right|$ by $x_{i}^{+}+x_{i}^{-}$to arrive at a standard $(\mathrm{QP})$.

The unique global minimum of this convex optimization problem possibly attained on a convex set can thus be obtained using standard algorithms available from any scientific program library. This criterion and similar ones have been considered for a while now [5], [8], [9].

To assess the role played by $h$ in the criterion (QP), it is useful to present the following dual of (QP) (DQP):

$$
\min _{x}\|A x\|_{2}^{2} \text { subject to }\left\|A^{T}(A x-b)\right\|_{\infty} \leq h
$$

(DQP)

where $\|x\|_{\infty}=\max _{i}\left|x_{i}\right|[10]$. The constraint of the dual says that at a feasible point, the residuals or reconstruction errors defined as $r=$ $b-A x$ are such that their correlations with the columns of $A$ never exceed $h$. The parameter $h$ allows to tune the maximal magnitude of these correlations. If the $\ell_{2}$ norm of the columns of $A$ is equal to one, this criterion allows thus for reconstruction errors that are of order $h$.

One can make the following remarks about the optimum $x^{*}$ of (QP) or (DQP) as $h$ varies from 0 to $+\infty$ : they are either obvious or can be deduced from the results presented in Sections III and VI.

$\diamond$ For $h=0$, one is left with $\min _{x}\|b-A x\|_{2}^{2}$, and, provided $A$ is full rank, the value of the minimum is zero and it is attained for all points in a convex set (a linear manifold).

$\diamond$ For $h=0^{+}$, i.e., for $h$ positive and arbitrarily close to zero, the solution is attained at the point(s) in the previous set having least $\ell_{1}$-norm. 
$\diamond$ For $h \geq\left\|A^{T} b\right\|_{\infty}$, the optimum is attained at $x^{*}=0$, see (DQP).

As will be shown later for $h=0^{+},(\mathrm{QP})$ has the same solution(s) as (LP). As $h$ increases, the optimum of (QP) drifts away from the optimum of (LP), $\|A x-b\|_{2}^{2}$, which was equal to zero, increases, and $\|x\|_{1}$ decreases. More generally, the interval $] 0^{+},\left\|A^{T} b\right\|_{\infty}[$ can be divided into subintervals characterized by the fact that within each such subinterval, the number of nonzero components of the optimum of (QP) is constant. As $h$ increases within one of the subintervals, the nonzero components of the optimum mostly decrease in absolute value until one of them becomes zero and $h$ hits the boundary of the subinterval. For $h \geq\left\|A^{T} b\right\|_{\infty}$, all the nonzero components have disappeared and the optimum remains at zero.

\section{OPTIMALITY CONDITIONS FOR (QP)}

In this section, we specify the necessary and sufficient conditions (NSC) satisfied at a minimum point of (QP) together with a sufficient condition for the minimum point to be unique.

The optimality conditions for $(\mathrm{QP})$ can be obtained in a quite traditional way by first transforming the problem into a quadratic program as indicated above and writing the first-order necessary (Kuhn-Tucker) conditions that are also sufficient since the problem is convex. We adopt a more direct path. Since the criterion is nonsmooth at zero because of the presence of $\|x\|_{1}$, we introduce the subdifferential of $\|x\|_{1}$, a set of vector called the subgradients, denoted $\partial\|x\|_{1}$

$$
\begin{aligned}
\partial\|x\|_{1} & =\left\{u \mid u^{T} x=\|x\|_{1},\|u\|_{\infty} \leq 1\right\} \\
& =\left\{u \mid u_{i}=\operatorname{sign}\left(x_{i}\right), \text { if } x_{i} \neq 0 \text { and }\left|u_{i}\right| \leq 1 \text { otherwise }\right\}
\end{aligned}
$$

where $\operatorname{sign}\left(x_{i}\right)=1$ when $x_{i}>0$ and $\operatorname{sign}\left(x_{i}\right)=-1$ when $x_{i}<0$. An NSC for $x^{*}$ to be a global minimum of (QP) is that the vector zero is a subgradient of the criterion at $x^{*}$ [7]

$$
\exists u \in \partial\|x\|_{1} \text { such that } A^{T}\left(A x^{*}-b\right)+h u=0 .
$$

To write this (NSC) in a more usable way, we distinguish between the nonzero components and the zero components of $x^{*}$. We denote $\bar{x}^{*}$ the reduced dimensional vector built upon the nonzero components of $x^{*}$. Similarly, $\bar{A}$ denotes the associated columns in $A$. One then has, e.g., $A x^{*}=\bar{A} \bar{x}^{*}$. For the rows in (NSC) associated with the nonzero entries $\bar{x}^{*}$, the subgradient is unique and equal to the gradient, for the other rows the subgradient takes any value in $[-1,1]$. The necessary and sufficient conditions become

$$
\begin{aligned}
\bar{A}^{T}\left(b-\bar{A} \bar{x}^{*}\right) & =h \operatorname{sign}\left(\bar{x}^{*}\right) \\
\left|a_{j}^{T}\left(b-\bar{A} \bar{x}^{*}\right)\right| & \leq h, \quad \text { for } a_{j} \notin \bar{A} .
\end{aligned}
$$

One can further establish that if $\bar{A}$ is full rank and if the inequalities in $\left(\mathrm{NSC}_{2}\right)$ are strictly satisfied, then $x^{*}$ is the unique or strict minimum point of $(\mathrm{QP})$. The following theorem thus holds.

Theorem 1: Sufficient conditions for $x^{*}$ to be a strict minimum point of $(\mathrm{QP})$ are

$$
\begin{aligned}
& \text { 1) } \bar{A}^{T}\left(b-\bar{A} \bar{x}^{*}\right)=h \operatorname{sign}\left(\bar{x}^{*}\right) \\
& \text { 2) }\left|a_{j}^{T}\left(b-\bar{A} \bar{x}^{*}\right)\right|<h, \quad \text { for } a_{j} \notin \bar{A} \\
& \text { 3) } \bar{A} \text { full rank }
\end{aligned}
$$

where $\bar{x}^{*}$ denotes the reduced dimensional vector built upon the nonzero components of $x^{*}$ and $\bar{A}$ is such that $A x^{*}=\bar{A} \bar{x}^{*}$.
If the conditions of Theorem 1 are satisfied, (3) leads to

$$
\bar{x}^{*}=\bar{A}^{+} b-h\left(\bar{A}^{T} \bar{A}\right)^{-1} \operatorname{sign}\left(\bar{x}^{*}\right)
$$

with $\bar{A}^{+}=\left(\bar{A}^{T} \bar{A}\right)^{-1} \bar{A}^{T}$, the pseudoinverse of $\bar{A}$. The last term in the right-hand side of (5) is a bias term induced by the regularization term in (QP) which can be removed a posteriori if desired.

Relation (5) is an implicit equation since $\bar{x}^{*}$ appears on both sides. It is valid for a fixed $h$. However, provided there are no zero components in $\bar{A}^{+} b$, there exists an $h_{m}>0$ such that for $\left.h \in\right] 0, h_{m}$ [ taking $\operatorname{sign}\left(\bar{x}^{*}\right)=\operatorname{sign}\left(\bar{A}^{+} b\right)$ in the right-side of (5) leads to an $\bar{x}^{*}$ that satisfies (3). For $h$ sufficiently small, the choice of $\bar{A}$ somehow fully characterizes $\bar{x}^{*}$.

\section{SEPARABILITY CONDITION}

We are now ready to develop a sufficient condition under which a solution of $A x=b$ can be recovered from the unique minimum point of (QP). Let us denote $x_{o}$ the solution one wants to recover, let $\bar{x}_{o}$ be the $p$-dimensional vector built upon the nonzero component in $x_{o}$, and $\bar{A}_{o}$ the $(n, p)$-dimensional matrix built with the associated columns of $A$ so that $A x_{o}=\bar{A}_{o} \bar{x}_{o}=b$. We assume $\bar{A}_{o}$ to be full rank.

For a nonzero $h$, the optimum of (QP) is attained at a point, we denote $x^{*}$, that achieves a compromise between the two parts of the criterion $\|A x-b\|_{2}^{2}$ and $\|x\|_{1}$. One thus has $A x^{*} \neq b$ and $x^{*}$ is never exactly equal to a solution of $A x=b$. We will therefore consider that (QP) allows to recover $x_{o}$ if its optimum $x^{*}$ and $x_{o}$ have their nonzero components at the same locations and with the same signs.

Theorem 2: The solution $x_{o}$ of $A x=b$ with $\bar{A}_{o}$ and $\bar{x}_{o}$ as defined above and $\bar{A}_{o}$ a full rank matrix, can be recovered from the unique optimum point of (QP) if

1) $\quad\left|a_{j}^{T} d_{o}\right|<1, \quad \forall a_{j} \notin \bar{A}_{o} \quad$ with $d_{o}=\bar{A}_{o}^{+T} \operatorname{sign}\left(\bar{x}_{o}\right)$

2) $h \in] 0, h_{m}[$ the domain in which

$$
\operatorname{sign}\left\{\bar{x}_{o}-h\left(\bar{A}_{o}^{T} \bar{A}_{o}\right)^{-1} \operatorname{sign}\left(\bar{x}_{o}\right)\right\}=\operatorname{sign}\left(\bar{x}_{o}\right)
$$

with $\bar{A}^{+}=\left(\bar{A}^{T} \bar{A}\right)^{-1} \bar{A}^{T}$, the pseudoinverse of $\bar{A}$.

Proof: We associate with $\bar{x}_{o}$ a vector $\bar{x}^{*}$ that, together with $\bar{A}_{o}$, satisfies the sufficient conditions of Theorem 1 . Since $\bar{x}_{o}=\bar{A}_{o}^{+} b$, the parameter $h$ in (7) is such that

$$
\operatorname{sign}\left(\bar{A}_{o}^{+} b\right)=\operatorname{sign}\left\{\bar{A}_{o}^{+} b-h\left(\bar{A}_{o}^{T} \bar{A}_{o}\right)^{-1} \operatorname{sign}\left(\bar{A}_{o}^{+} b\right)\right\}
$$

and the vector $\bar{x}^{*}$ defined as

$$
\bar{x}^{*}=\bar{A}_{o}^{+} b-h\left(\bar{A}_{o}^{T} \bar{A}_{o}\right)^{-1} \operatorname{sign}\left(\bar{x}^{*}\right), \quad \text { with } \operatorname{sign}\left(\bar{x}^{*}\right)=\operatorname{sign}\left(\bar{x}_{o}\right)
$$

then satisfies (3) with $\bar{A}$ replaced by $\bar{A}_{o}$. But for this vector $\bar{x}^{*}$ one has

$$
\left|a_{j}^{T}\left(b-\bar{A}_{o} \bar{x}^{*}\right)\right|=h\left|a_{j}^{T} \bar{A}_{o}^{+T} \operatorname{sign}\left(\bar{x}_{o}\right)\right|=h\left|a_{j}^{T} d_{o}\right|
$$

and it follows that (6) is equivalent to (4). This completes the proof since the vector $\bar{x}^{*}$ defined above, together with $\bar{A}_{o}$, satisfies all the conditions of Theorem 1 and allows to recover $x_{o}$.

While the size $h_{m}$ of the allowed domain for $h$ depends upon the magnitudes of the components of $\bar{x}_{o}$, condition (6) is independent of these magnitudes. Condition (6) defines two parallel separating hyperplanes $H_{ \pm}$, associated with a single vector $d_{o}, H_{ \pm}=\left\{a \mid a^{T} d_{o}=\right.$ $\pm 1\}$ which must be such that the columns that are present in the decomposition lie in these hyperplanes: $\left|a_{j}^{T} d_{0}\right|= \pm 1, \forall a_{j} \in \bar{A}_{o}$ (see the definition of the vector $d_{o}$ ), while the other columns (not in $\bar{A}_{o}$ ) must lie strictly in between (see (6)). 
This is why in [4], [5] we called (6) the separability condition.

\section{SPARSITY CONDITION}

In this section, we specialize the separability condition (6) to the specific problem considered in [1], [2] and assume the columns $a_{j}$ of $A$ to have unit Euclidean norm. We transform the separability condition (6) into the more usable but probably more conservative condition (2). We prove the following.

Theorem 3: If the columns in $A$ are normalized to one in $\ell_{2}$ norm, then $(2) \Rightarrow(6)$, i.e.,

$$
\begin{aligned}
\left\|x_{o}\right\|_{0}<\frac{1}{2}\left(1+\frac{1}{M}\right) \Rightarrow\left|a_{j}^{T} \bar{A}_{o}\left({\overline{A_{o}}}^{T} \bar{A}_{o}\right)^{-1} \operatorname{sign}\left(\overline{x_{o}}\right)\right|<1, \\
\forall a_{j} \notin \overline{A_{o}} .
\end{aligned}
$$

Proof: For simplicity we set $p=\left\|x_{o}\right\|_{0}$. Remember that

$$
M=\sup _{1 \leq i \neq j \leq m}\left|a_{i}^{T} a_{j}\right| \text {. }
$$

The diagonal part of ${\overline{A_{o}}}^{T} \bar{A}_{o}$ is equal to the identity matrix $I$ since we assume $a_{j}^{T} a_{j}=1, \forall j$. We write $\bar{A}_{o}{ }^{T} \bar{A}_{o}=I-H$, where $H$ is built with the nondiagonal elements of $-{\overline{A_{o}}}^{T} \bar{A}_{o}$.

Since all the nonzero components in the square matrix $H$ of order $p$ are smaller than $M$, its spectral radius $\rho(H)$ satisfies $\rho(H) \leq(p-$ 1) $M$ by Gershgorin theorem [11]. From (2) it thus follows that $\rho(H)<$ 1 and one can apply Neumann's lemma [11] to get

$$
\left({\overline{A_{o}}}^{T} \overline{A_{o}}\right)^{-1}=(I-H)^{-1}=I+\sum_{k>0} H^{k} .
$$

For a matrix $B$, we denote $|B|$ the matrix with entries $\left|b_{i, j}\right|$ and write $B<C$ if $b_{i, j}<c_{i, j}, \forall i, j$, one then has

$$
\begin{aligned}
\left|\left(\overline{A_{o}} \bar{A}^{T}\right)^{-1}\right| & =\left|I+\sum_{k>0} H^{k}\right| \leq I+\sum_{k>0}\left|H^{k}\right| \\
& \leq I+\sum_{k>0}|H|^{k} \leq I+\sum_{k>0}(M E)^{k} \\
& =(I-M E)^{-1}
\end{aligned}
$$

where $E$ is a square matrix whose entries are equal to one except those on the diagonal which are equal to zero. One thus has $E=\mathbf{1}-\boldsymbol{I}$, where 1 denotes here and below a matrix of ones with adequate dimensions. It is then easy to check that

$$
\begin{aligned}
& I-M E=(1+M) I-M \mathbf{1} \Rightarrow(I-M E)^{-1}=\alpha I+\beta \mathbf{1}, \\
& \text { with } \alpha=\frac{1}{1+M} \text { and } \beta=\frac{M}{(1+M)(1+M-M p)} .
\end{aligned}
$$

Combining this result with, e.g., $\left|a_{j}^{T} \bar{A}_{o}\right| \leq M \mathbf{1}^{T}$ leads to the following inequalities:

$$
\begin{aligned}
\left|a_{j}^{T} \overline{A_{o}}\left(\overline{A_{o}}{ }^{T} \overline{A_{o}}\right)^{-1} \operatorname{sign}\left(\overline{x_{o}}\right)\right| & \leq\left|a_{j}^{T} \overline{A_{o}}\right|\left|\left(\overline{A_{o}}{ }^{T} \overline{A_{o}}\right)^{-1}\right| \\
& \times\left|\operatorname{sign}\left(\overline{x_{o}}\right)\right| \\
& \leq M \mathbf{1}^{T}\left|\left(\overline{A_{o}}{ }^{T} \overline{A_{o}}\right)^{-1}\right| \mathbf{1} \\
& \leq M\left(\alpha p+\beta p^{2}\right) \\
& \leq \frac{M p}{1+M-M p}<1
\end{aligned}
$$

where the very last step follows from (2).

Combining Theorems 2 and 3, we have established the following.
Corollary: If a solution $x_{o}$ of $A x=b$ satisfies (2), then (QP) has a unique solution point from which $x_{o}$ can be recovered, provided $h$ in (QP) has been taken small enough.

Indeed, taking $h=0^{+}$guarantees that $x^{*}=x_{o}$ as is the case for the (LP) criterion.

Remark: Specializing the preceding proof to the case where $A$ is the concatenation of two unitary matrices yields the condition $\|x\|_{0}<$ $(\sqrt{2}-0.5) / M$, obtained in [2], that is slightly weaker than (2). In this case, ${\overline{A_{o}}}^{T} \overline{A_{o}}$ has a block diagonal form and $\left|a_{j}^{T} \overline{A_{o}}\right|$ has some zero entries leading quite easily to this tighter bound.

\section{Special Case of the Linear Program}

We claimed in Section II that the optimum of (QP) converges to the optimum of (LP) when $h$ in (QP) decreases to zero. For simplicity, we assume both optima to be strict and we rewrite (5) as

$$
\bar{x}^{*}(h)=\bar{A}^{+} b-h\left(\bar{A}^{T} \bar{A}\right)^{-1} \operatorname{sign}\left(\bar{x}^{*}(h)\right)
$$

where we emphasize the dependence of $\bar{x}^{*}$ on $h$. We assume that $h \in$ ]0, $h_{m}$ [, the nonempty domain in which $\operatorname{sign}\left(\bar{x}^{*}(h)\right)=\operatorname{sign}\left(\bar{A}^{+} b\right)$. In this domain, $(\mathrm{QP})$ is equivalent to

$$
\min _{x}\|x\|_{1} \text { subject to }\|A x-b\|_{2}^{2} \leq B(h)
$$

with $B(h)=\left\|A x^{*}(h)-b\right\|_{2}^{2}=0\left(h^{2}\right)$. But the optimum point of this new problem converges to the optimum of (LP) as $h \downarrow 0$ since $B(h) \downarrow$ 0 . This establishes the claimed equivalence and actually establishes that condition (2) is also sufficient for (LP) to retrieve the unique sparsest solution.

A direct analysis of this result can indeed be achieved quite easily along the lines used for the $(\mathrm{QP})$ criterion. Let us do so for completeness in this section using the notations introduced earlier.

Theorem 4: The solution $x_{o}$ of $A x=b$, with $b=A x_{o}=\bar{A}_{o} \bar{x}_{o}$ and $\bar{A}_{o}$ a full rank matrix, is the unique optimum point of (LP) if

$$
\left|a_{j}^{T} d_{0}\right|<1 \forall a_{j} \notin \bar{A}_{0} \text { for some } d_{0} \text { such that } \bar{A}_{0}^{T} d_{0}=\operatorname{sign}\left(\bar{x}_{o}\right) \text {. }
$$

Proof: Let us recall the (LP) criterion

$$
\min _{x}\|x\|_{1} \text { subject to } A x=b .
$$

A slight difficulty arises from the fact that the expected solution $x_{o}$ of (LP) is degenerate, i.e., has less than $n$ nonzero components. In order to characterize the optimality of the solution, it is then convenient to introduce the dual linear program [6]

$$
\max _{d} d^{T} b \text { subject to }\left\|d^{T} A\right\|_{\infty} \leq 1
$$

which, as a linear program, is not in standard form either.

For a vector $x_{o}$ satisfying $A x_{o}=\bar{A}_{o} \bar{x}_{o}=b$ to be an optimum of (LP), one needs to be able to associate with it a solution, say $d_{o}$, of (DLP) that is feasible and has an identical cost

$$
\left\|d_{o}^{T} A\right\|_{\infty} \leq 1 \text { and }\left\|x_{o}\right\|_{1}=d_{o}^{T} b .
$$

Rewriting the primal cost $x_{o}^{T} \operatorname{sign}\left(x_{o}\right)=\bar{x}_{o}^{T} \operatorname{sign}\left(\bar{x}_{o}\right)$ and the dual $\operatorname{cost} d_{o}^{T} b=d_{o}^{T} A x_{o}=d_{o}^{T} \bar{A}_{o} \bar{x}_{o}$ shows that they are equal for all $\bar{x}_{o}$ if $d_{o}^{T} \bar{A}_{0}=\operatorname{sign}\left(\bar{x}_{o}^{T}\right)$. For $d_{o}$ to be a feasible point of (DLP) it remains then to check

$$
\left|a_{j}^{T} d_{o}\right| \leq 1, \quad \forall a_{j} \notin \bar{A}_{o} .
$$

For $x_{o}$ to be the unique solution of (LP) one has to ask in addition that the dual problem be nondegenerate in the sense that all constraints 
associated with zero Lagrange multipliers be strictly satisfied. Since the Lagrange multipliers of (DLP) are the components of $x_{o}$, this means that the inequalities in (10) have to be strict, transforming (10) into (9).

From Theorems 3 and 4, we have the following.

Corollary: If (2) holds then $x_{o}$ is the unique solution of (LP).

Condition (9) is weaker than (6) that needs to hold for (QP), since the vector $d_{o}$ is now less constrained. It belongs to an $(n-p)$-dimensional linear manifold defined in (9) while the vector $d_{o}$ defined in (6) for the (QP) criterion is a specific vector of this manifold, the one of least $\ell_{2}$ norm. It is indeed possible to construct toy examples where (LP) allows to recover a solution (not satisfying the sufficient condition (6)) while (QP) does not.

\section{CONCLUSION}

Given any $(n, m)$ matrix $A$ with $m>n$ and a solution $x_{o}$ of the set of under-determined linear equations $A x=b$, we have given sufficient conditions (6) under which it is possible to recover $x_{0}$ from the optimum of a quadratic program [5] and slightly weaker sufficient conditions (9) under which this is possible using a linear program.

If the columns in $A$ are further taken to have unit Euclidean norm and one is primarily interested in sparse solutions then we have shown that both (6) and (9) are satisfied if

$$
\left\|x_{o}\right\|_{0}<\frac{1}{2}\left(1+\frac{1}{M}\right), \quad \text { with } M=\sup _{1 \leq i \neq j \leq m}\left|a_{i}^{T} a_{j}\right|
$$

where $\|x\|_{0}$ is the number of nonzero components in $x$. This also establishes that this condition guarantees that $x_{o}$ is the unique sparsest solution to $A x=b$. This result on sparse solutions extends those previously published which considered $(n, 2 n)$ matrices $A$ obtained by concatenating two unitary matrices and is similar to the result obtained in [3] using a completely different approach.

The result holds both for the linear program (LP) seeking the minimal $\ell_{1}$ norm solution to $A x=b$ and for the following quadratic program parametrized by an hyper-parameter $h$ :

$$
\min _{x} \frac{1}{2}\|A x-b\|_{2}^{2}+h\|x\|_{1}, \quad h>0
$$

For the criterion (QP), under an additional condition on $h$ that is always satisfied for $h=0^{+}$, its unique optimum $x^{*}$ and the sought-for solution $x_{o}$ have their nonzero components at the same locations and with identical signs. Since this criterion allows for nonzero residuals $r=A x^{*}-b \neq 0$, with magnitudes of the order of $h, x^{*}$ is never a solution of $A x=b$ and is the best one can expect.

Allowing for nonzero residuals is of practical importance since it allows for the presence of noise or errors due to mismodeling. It is also the main reason for considering $(\mathrm{QP})$ rather then $(\mathrm{LP})$. In the presence of noise, the true model is

$$
b=A x_{o}+e
$$

with $e$ a vector of white Gaussian noise, for instance. Asking for an exact reconstruction of $b$ as a linear combination of columns of $A$ will yield a nonsparse estimate $x^{*}$ of $x_{o}$. One possibility is then to threshold the components of this estimate in order to recover $x_{o}$ by removing the small components induced by the presence of the noise. But a more sensible approach is probably to take into account from the beginning the presence of the noise and to solve (QP) with $h$ of the order of the standard deviation of the noise [9], [10] to get a sparse $x^{*}$ that does not require pruning.

Another similar situation arises when the true model of the observed vector $b$ is

$$
b=\sum_{i=1}^{p} \alpha_{i} a\left(\tau_{i}\right)
$$

with $a(\tau)$ a known family of vectors parametrized by a scalar $\tau$ and $\alpha_{i}$ the scalar weights. In order to apply the previous setting to this estimation problem, one way to proceed is to uniformly discretize the values of $\tau$ over its compact domain to get the $m$ columns $a_{j}=\left.a(\tau)\right|_{\tau=t_{j}}$ of the $A$ matrix. Since the $\tau_{i}$ will generically not belong to the sampling points set $\left\{t_{j}\right\}$, there is again no sparse representation of $b$ as a linear combination of the columns of $A$. In the simplest case of a single component $b=a\left(\tau_{1}\right)$, the components of $x^{*}$ can be shown to be samples from an interpolating function [13].

Tentative analyses of these more complex scenarios, where both noise and mismodeling errors are present, can be found in [12], [13] for $(\mathrm{QP})$ or the following modified (LP) criterion:

$$
\min _{x}\|x\|_{1} \text { subject to }\|A x-b\|_{\infty} \leq \rho .
$$

\section{ACKNOWLEDGMENT}

The author wants to thank one of the reviewers and the Associate Editor for the numerous comments they made that helped improve the readability of the submission.

\section{REFERENCES}

[1] D. L. Donoho and X. Huo, "Uncertainty principles and ideal atomic decomposition," IEEE Trans. Inform. Theory, vol. 47, pp. 2845-2862, Nov. 2001.

[2] M. Elad and A. M. Bruckstein, "A generalized uncertainty principle and sparse representation in pairs of bases," IEEE Trans. Inform. Theory, vol. 48, pp. 2558-2567, Sept. 2002.

[3] R. Gribonval and M. Nielsen, "Sparse representations in unions of bases," IEEE Trans. Inform. Theory, vol. 49, pp. 3320-3325, Dec. 2003.

[4] J. J. Fuchs, "Une approche à l'estimation et l'identification simultanées," in XVIe Colloque GRETSI, vol. 2, Grenoble, France, 1997, pp. $1273-1276$.

[5] — , "Detection and estimation of superimposed signals," in Proc. IEEE Int. Conf. Acoustics, Speech, and Signal Processing (ICASSP), vol. III, Seattle, WA, 1998, pp. 1649-1652.

[6] D. G. Luenberger, Introduction to Linear and Nonlinear Programming. Boston, MA: Addison Wesley, 1973.

[7] R. Fletcher, Practical Methods of Optimization. New York: Wiley, 1987.

[8] S. Chen, D. Donoho, and M. Saunders, "Atomic decomposition by basis pursuit,” SIAM J. Scient. Comput., vol. 20, no. 1, pp. 33-61, Jan. 1999.

[9] J. J. Fuchs, "Multipath time-delay detection and estimation," IEEE Trans. Signal Processing, vol. 47, pp. 237-243, Jan. 1999.

[10] _ - "On the application of the global matched filter to DOA estimation with uniform circular arrays," IEEE Trans. Signal Processing, vol. 49, pp. 702-709, Apr. 2001

[11] A. S. Householder, The Theory of Matrices in Numerical Analysis. New York: Blaisdell, 1964.

[12] J. J. Fuchs, "Extension of the Pisarenko method to sparse linear arrays," IEEE Trans. Signal Processing, vol. 45, pp. 2413-2421, Oct. 1997.

[13] J. J. Fuchs and B. Delyon, "Minimum $L_{1}$-norm reconstruction function for oversampled signals: Application to time-delay estimation," IEEE Trans. Inform. Theory, vol. 46, pp. 1666-1673, July 2000. 